\title{
BMJ Open Duration of sick leave after same-day discharge for lower extremity arterial disease and varicose vein interventions in active population of French patients, 2013-2016: observational study
}

To cite: Hamid A, Lamirault G, Gouëffic Y, et al. Duration of sick leave after sameday discharge for lower extremity arterial disease and varicose vein interventions in active population of French patients, 2013-2016: observational study. BMJ Open 2020;10:e034713. doi:10.1136/ bmjopen-2019-034713

- Prepublication history and additional material for this paper are available online. To view these files, please visit the journal online (http://dx.doi. org/10.1136/bmjopen-2019 034713).

Received 02 October 2019 Revised 14 February 2020 Accepted 28 May 2020

\section{Check for updates}

(C) Author(s) (or their employer(s)) 2020. Re-use permitted under CC BY-NC. No commercial re-use. See rights and permissions. Published by BMJ.

${ }^{1}$ Univ Rennes, EHESP, REPERES (Recherche en Pharmacoépidémiologie et Recours aux Soins) - EA 7449, EHESP, Rennes, France

${ }^{2}$ Institut du thorax, INSERM, CNRS, UNIV Nantes, Nantes, France

${ }^{3}$ Institut du thorax, CHU Nantes, Nantes, France

${ }^{4}$ Vascular Center, Groupe hospitalier Paris Saint-Joseph, Paris, France

Correspondence to

Dr Nolwenn Le Meur; nolwenn.lemeur@ehesp.Fr

\section{ABSTRACT}

Objective To assess whether disparities in rates of sameday discharge for lower extremities arterial disease $(5 \%)$ and varicose vein interventions $(90 \%)$ are associated with the burden of postprocedural rehabilitation process, measured through the duration of sick leave.

Design Retrospective observational study using French National Health Insurance data in 2012-2016.

Setting The French National Health Data System (Système National des Données de Santé), which covers $98.8 \%$ of the 66 million people in the French population. Participants French workforce population aged 18 to 65 years old who underwent a first angioplasty with stent placement for lower extremities arterial disease (LEAD, $n=30238)$ or a first varicose vein intervention $(n=265670)$ between 2013 and 2016.

Main outcome measures Duration and renewals of sick leave within 180 days after endovascular intervention, continuity of care and prescription indices to assess coordination among healthcare professionals after intervention associated with specific intervention settings: conventional (inpatient) or same-day discharge (outpatient). Association was estimated by multivariate negative binomial regressions adjusting for age, gender and comorbidities.

Results Outpatient settings decrease the incidence rate ratio (IRR) of the number of cumulated days of sick leave by $14 \%$ in both interventions. The increasing variety of prescribers decreases the IRR of cumulated days of sick leave and prescription renewals for varicose interventions by $25 \%$ and $21 \%$, respectively, but increases them for LEAD interventions by $240 \%$ and $106 \%$. Less coordination between healthcare specialists increases the IRR of cumulative days of sick leave and renewals by $37 \%$ and $29 \%$ for varicose, and $11 \%$ and $9 \%$ for LEAD interventions. Conclusions Low rates of outpatients in LEAD angioplasty does not seem related to the duration of sick leave. Outpatient setting reduces the duration of sick leave and their renewals, whatever the intervention. Coordination of healthcare professionals is a key element of interventions follow-up with pathology specificities.

\section{INTRODUCTION}

In the French healthcare system, same-day discharge procedures are outpatients'
Strengths and limitations of this study

- This retrospective study is based on data extracted from the French National Health Insurance System (SNDS-Système National des Données de Santé) which covers $98.8 \%$ of the 66 million people in the French population.

- The cohort enables the analysis of healthcare pathways including hospital stays, office medicine consultations, drug prescriptions and sick leave prescriptions.

- The cohort includes patients who beneficiated from an angioplasty with stent placement which represents $85 \%$ of the angioplasties for lower extremities arterial disease.

- The SNDS lack of detailed sociodemographic and clinical information preventing the inclusion of valuable information relating to hospital stay conversion, family environment and socioprofessional category in our analysis model.

hospitalisations, while inpatients' care include overnight hospitalisations. Outpatient procedure appears to be beneficial for both patients' quality of care and healthcare systems by relieving the pressure on hospital beds and material resources. ${ }^{1-3}$ Long time lagging behind for the development of ambulatory surgeries and interventions, France has recently made significant progress in developing same-day discharge. Since 2010, same-day discharge has grown up to represent on average $56 \%$ of all surgeries and interventions but, depending on the medical procedure and geographical area, important variations can be observed. In 2016, almost $90 \%$ of varicose vein interventions were performed in outpatient setting, while we estimated at $<5 \%$ the rate of same-day discharge procedures for stent placement in the context of lower extremities arterial disease (LEAD), with regional variations from $0 \%$ to $58 \%$. 
These disparities may result from the complexity of the therapeutic management for patients with LEAD, who tend to have more complicated postprocedural follow-up and rehabilitation than patients with varicose vein as they are older, they have more comorbidities, and their condition is more disabling. As a consequence, outpatient endovascular procedures for patients with LEAD may result in shifting the burden of early postprocedural follow-up to ambulatory health services and in increasing the time before resuming work. ${ }^{4}$

To approximate the burden of postprocedural rehabilitation in the context of inpatient and outpatient settings, we propose to compare the number of days of medical leave from work and the number of their renewals after intervention. Research conducted on sick leave according to intervention settings for the treatment of varicose vein and angioplasty with stent placement for LEAD is scarce. In varicose veins, most studies compare procedures (ie, stripping vs radiofrequency or laser ablation) but not the setting as most interventions are day cases. However, few international studies report that the mean number of days of sick leave after conventional surgery of varicose vein vary between 4 and 14 days, whatever the intervention setting. The French guidelines by the health regulatory agency vary from 3 days to 10 days (including the day of the procedure) in the case of sedentary workers. ${ }^{5}$ For LEAD procedures, the French guidelines concern only inpatient interventions and vary from 7 days to 28 days, according to the type of intervention and the patient's employment characteristics. ${ }^{5}$
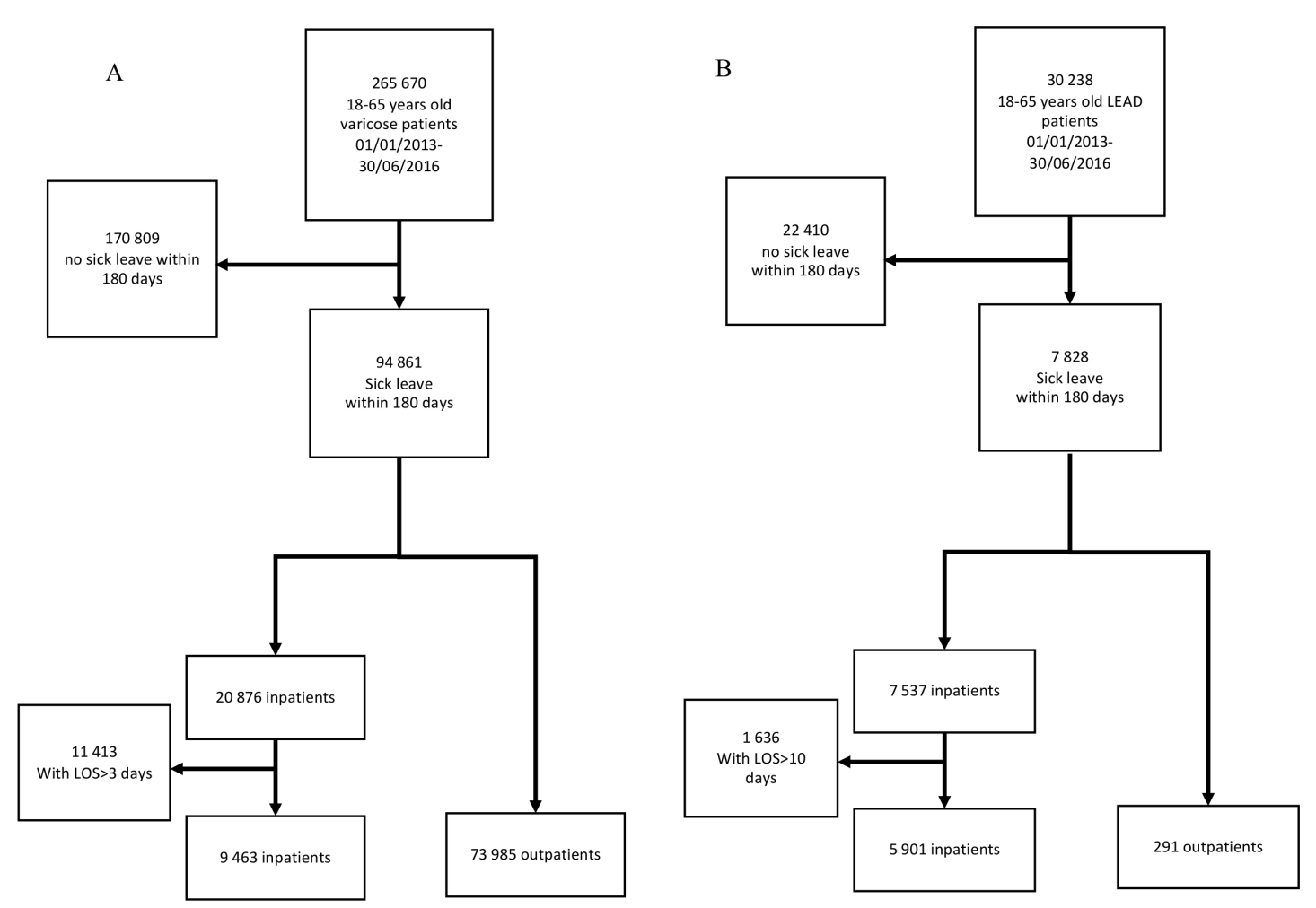

Figure 1 Flowchart of study population. (A) Patients with varicose vein. (B) Patients with LEAD. LEAD, lower extremity arterial disease; LOS, Length of stay in hospital.

In this paper, we propose for the first time a description and a comparison of duration of sick leave in function of the hospitalisation setting (ie, inpatient or outpatient) after LEAD and varicose interventions. The indicators used are (1) the delays before resuming work and (2) the number of renewals of sick leave prescriptions per patient. The differences in sick leave duration and renewals according to healthcare prescribers in the continuum of care are also highlighted.

\section{METHODS}

A retrospective analysis from 1 January 2013 to 30 June 2016 was conducted using data from the French National Health Data System (Système National des Données de Santé, SNDS), which covers $98.8 \%$ of the 66 million people in the French population. The SNDS contains anonymous and individual health insurance users' data with demographic characteristics and all reimbursed care, including outpatient medical care, ambulatory consultations and hospitalisation discharge data. ${ }^{6}$

\section{Patient and public involvement \\ No patient involved.}

\section{Study population and demographics}

The population of interest was the French workforce population aged 18 to 65 years old. All incident patients who underwent angioplasty and stent placement for LEAD ( $n=30238)$ or varicose vein invasive interventions 
(ligature, stripping or crossectomy, $\mathrm{n}=265670$ ) between 1 January 2013 and 30 June 2016 were included (figure 1). Clinical selection criteria were based on hospital discharge principal diagnosis identified by International Classification of Diseases, 10th Revision codes and surgical procedures identified by the French Common Classification of Medical Procedures (online supplementary tables 1 and 2). Outpatient setting corresponded to 1 day intervention where patient stayed less than 12 hours in a hospital with same-day discharge, coming from home and leaving back home. For comparison purposes, and following health regulatory agencies' guidelines, inpatient whose hospital length of stay was longer than 10 days for an endovascular procedure and 3 days for a varicose veins' intervention were excluded (online supplementary figures 1 and 2). ${ }^{5}$ Patients with no sick leave within 180 days following the intervention were excluded. The final population included 83448 patients with varicose and 6192 patients with LEAD .

\section{Study variables and outcomes}

Each patient was characterised by the following covariates: gender, age categorised into three age groups (18-39, 40-59, 60-65), surgical intervention setting (inpatient or outpatient) and group of comorbidity index. The Charlson's Comorbidity Index was computed per patient to quantify the burden of comorbidities. ${ }^{78}$ Comorbidities were obtained based on the principal and associated diagnoses that a patient may have had during hospitalisation 1 year prior the index intervention. For regression analysis, the Charlson's Comorbidity Index was transformed into five classes $(0,[1-2], 3,4,[5-12])$.

For each patient, continuum of care after surgery was characterised by the Bice-Boxerman Continuity of Care Index (COCI), ${ }^{9} 10$ and an adapted version measured the continuity of sick leave prescribers (COPI, online Supplementary equations 1 and 2). The COCI varies between 0 and 1 , and measures the extent to which a patient seek care through a unique care provider category (eg, COCI=1 if only general practitioner (GP)) or several different ones (eg, COCI $\sim 0$ if GP+angiologists+...). The COPI varies between 0 and 1 , and measures the extent to which a patient had his/her sick leaves prescribed by a unique care provider category (COPI $=1$ ) or several different ones (COPI 0).

Categories of sick leave prescribers were built according to the medical specialties and place of practice: 'GP' refers to general practitioners, 'hospital' refers to prescriptions written by hospital services, 'specialists' refer to radiologists, vascular and cardiovascular doctors in town practice (ie, medical office) and 'others' refer to all other prescribers in medical offices.

To approximate the postprocedural rehabilitation process, the outcomes of the study were (1) the cumulative days off work for medical leave after hospital intervention per patient within 180 postprocedural days, and (2) the number of renewals of medical leave from work per patient within 180 postprocedural days and without rehospitalisation.

\section{Statistical analyses}

The patients' characteristics were described and compared using Pearson's $\chi^{2}$ test when qualitative and Student's t-test when quantitative. A $p$ value $<0.05$ was considered statistically significant. The association between covariates and outcomes of interest was assessed using negative binomial regression analyses. Propensity score weighting was used to address possible treatment bias associated with the year of intervention, sex, age, comorbidities and agexcomorbidity interactions. ${ }^{11}$ Results were reported as incidence rate ratios (IRRs) with 95\% CIs and $\mathrm{p}$ values. Forest plot was used to visually compare the results. All computations were performed using R. ${ }^{12}$

\section{RESULTS}

Patients with LEAD differ from patients with varicose vein by most characteristics (table 1). Only $4.7 \%$ of patients with LEAD were outpatients while it concerns $89 \%$ of patients with varicose vein. Among the patients with varicose vein, the female/male sex ratio approximated 1.5. Among patients with LEAD, the sex ratio was around 0.19 in both settings. More than $90 \%$ of patients with varicose vein had no comorbidity. Patients with LEAD were older than patients with varicose vein with most patients aged between 40 and 59 years old. Comorbidity profiles were different between pathologies with a majority of diabetes and pulmonary diseases in the population with varicose vein (online supplementary table 3 and figure 1) and a majority of cardiovascular diseases in the population with LEAD (online supplementary table 4 and figure 2).

\section{Varicose vein and postprocedural duration of sick leave}

An increase trend of IRR was observed for the cumulated number of workday breaks in relation with comorbidities level compared with no comorbidities, whatever the intervention setting. Similarly, an increase trend of IRR was estimated for the cumulated number of workday breaks and number of workday break renewals in relation with population ageing ( (figure 2 , online supplementary table 5 and 6). The mean duration of the first sick leave was similar for inpatients and outpatients with 3.90 and 3.87 days, respectively ( $p$ value $=0.52$ ). The average duration of each prescribed sick leaves over the 180 days period after intervention was significantly different between inpatients and outpatients with 5.95 days and 5.51 days, respectively ( $p$ value $<2 e-16$ ). On average, the cumulated number of workday breaks and the number of renewals of prescriptions differ between inpatients and outpatients with 61 days and 48.5 days of cumulated number of workday breaks, respectively and prescription renewals 8.7 and 7.2 times. Intervention settings have a significant impact on the cumulated number of workday breaks and the number of sick leave renewals. While holding all other variables constant, interventions in outpatient setting 
Table 1 Characteristics of the active population of French patients who had a sick leave prescription within 30 days of a vascular intervention for LEAD or varicose veins between 1 January 2013 and 30 June 2016

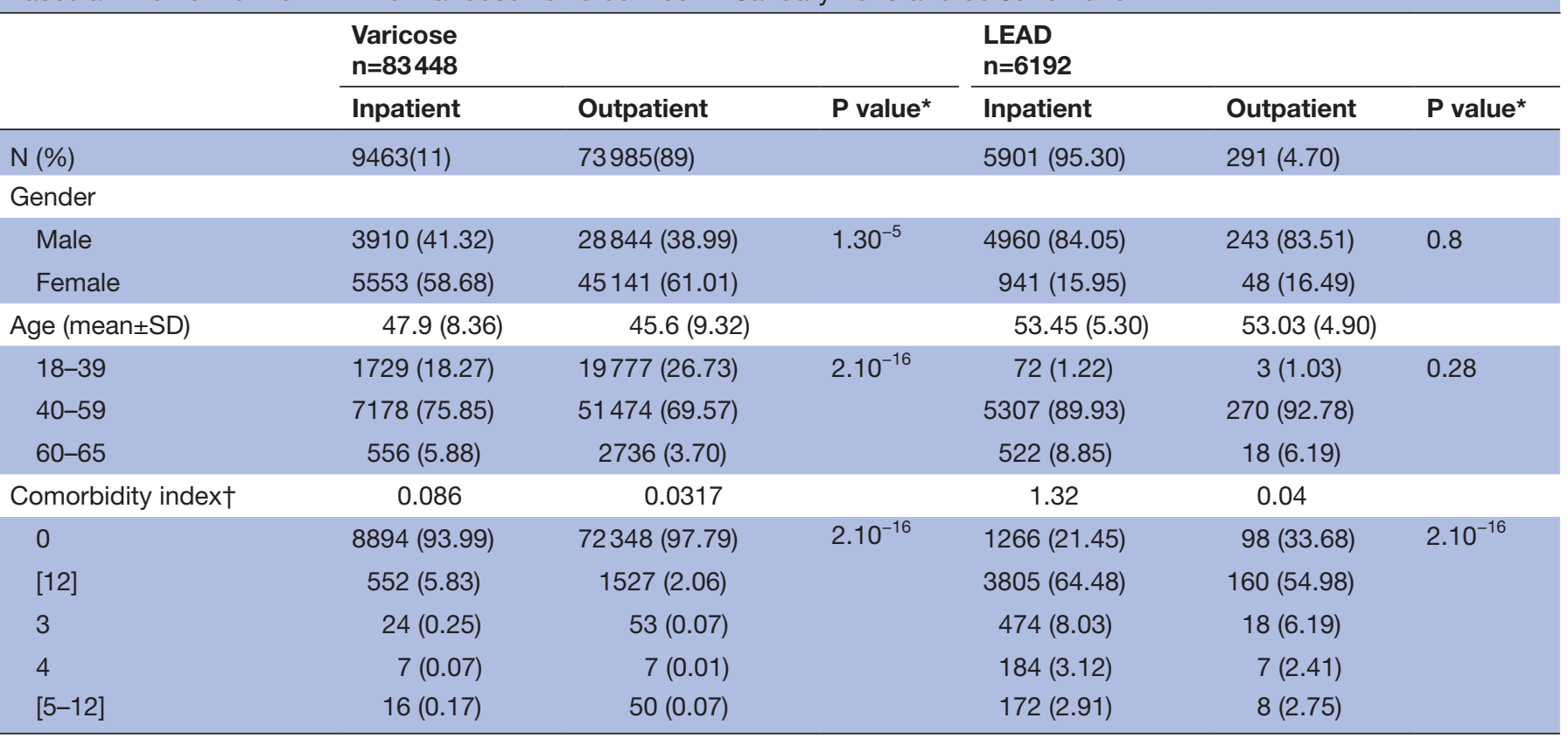

*P values are results of univariate tests: Fisher's exact test for qualitative variable and Student's t-test for quantitative variables. †As defined by Charlson's Comorbidity algorithm.

LEAD, lower extremity arterial disease.

significantly decreased the trend of IRR of the number of sick leave days by $14 \%$, and the IRR of sick leave renewals by $9 \%$ compared with inpatient settings.
Compared with sick leaves prescribed by GPs, the IRR of the total number of cumulated sick leave days prescribed within 180 days of varicose intervention decreased by $43 \%$

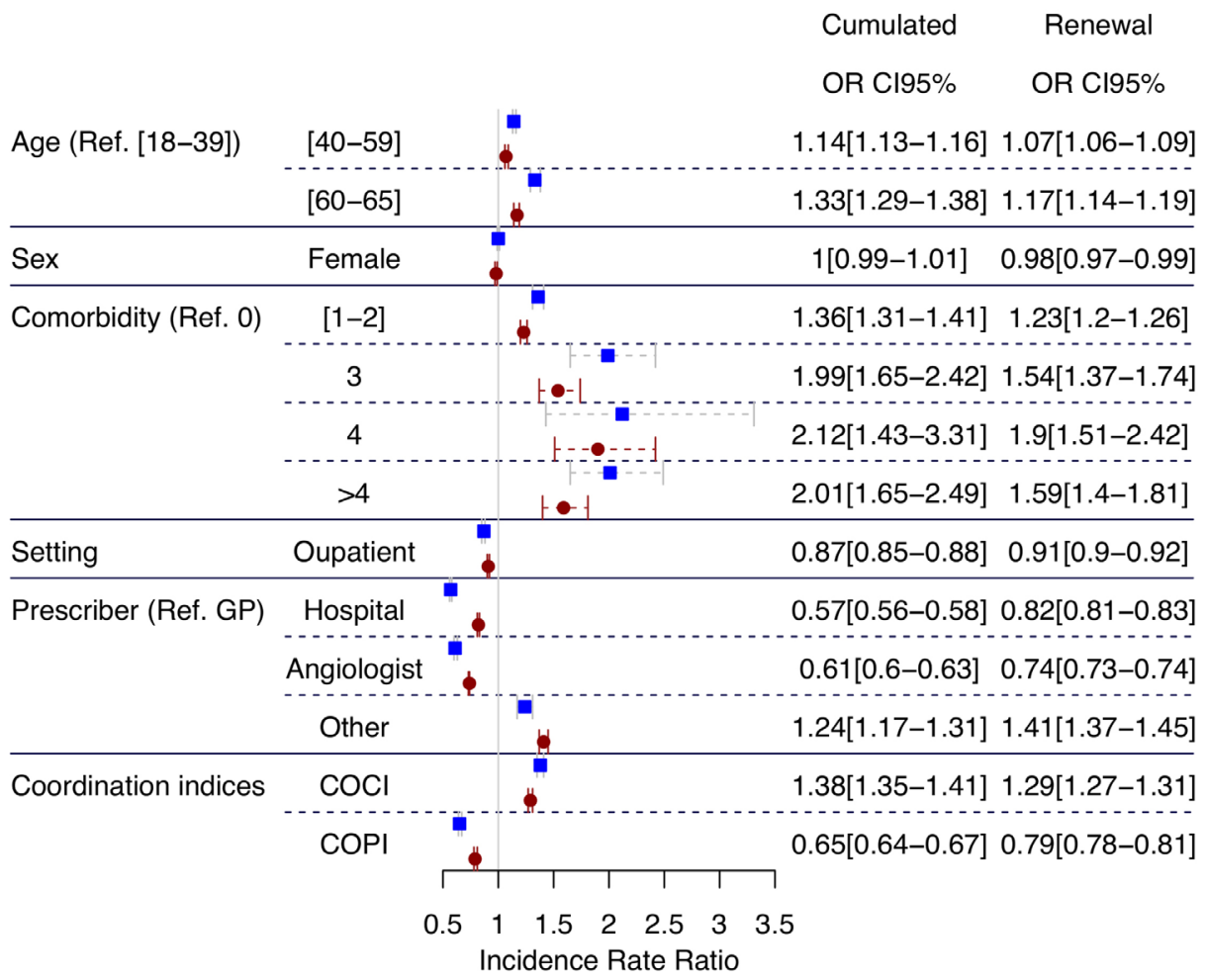

Figure 2 IRRs of cumulated and prescription renewals of sick leave after varicose vein intervention, in France between 2013 and 2016. $\mathrm{COCl}$, Continuity of Care Index; IRRs, incidence rate ratios. 
if prescribed within hospitals and by $39 \%$ if prescribed by vascular doctors in outpatient sites (eg, angiologists in medical offices) but increased by $40 \%$ if prescribed by other specialists in their medical offices. Similarly, the IRR of the total number of sick leave renewals was expected to decrease by $19 \%$ if prescribed in a hospital and by $27 \%$ if prescribed by a vascular specialist but increased by $47 \%$ if it was prescribed by another specialist.

The COCI showed that when the number of visits to the same group of providers increased, the IRR of cumulative days of sick leave increased by a factor of $38 \%$ and the number of sick leave renewals increased by a factor of 29\%. The COPI indicated that when the number of prescribers from distinct specialties decreases, the IRR of the cumulated days of sick leave decreased by $25 \%$, and the IRR the total number of sick leave renewals decreased by $21 \%$. Interestingly, outpatients have a lower percentage of visits to the same group of providers but a higher percentage of prescriptions from the same group of providers than inpatients, possibly synonymous of tighter coordination (online supplementary table 7). Although COCI and COPI indices are not linearly correlated $(\mathrm{r}=-0.05)$, when prescriptions were delivered by few providers of the same specialty, sick leaves were shorter on average, reducing the overall cumulated days of sick leaves or their renewals.

\section{LEAD and postprocedural duration of sick leave}

In the population with LEAD, the number of prescription renewals for workday breaks and their cumulated number did not seem to be significantly associated with the age and the sex of the patient (figure 3). However, the levels of comorbidities did affect the IRR of the cumulated number of workday breaks and the renewals of workday break prescriptions. Although no specific trend was observed, two groups could be highlighted: comorbidity levels 1 and 2 demonstrated an IRR increase of $12 \%$ for the cumulated number of workday breaks compared with no comorbidities and comorbidity levels above 2 showed an IRR increase of around $50 \%$. Although, the mean duration of the first sick leaves was similar whatever the intervention setting (5.29 and 5.46 days for inpatient and outpatient, respectively with $\mathrm{p}$ value $=0.57$ ), intervention settings was a significant determinant of the number of the cumulated workday breaks (figure 3) (online supplementary table 8). Outpatient management decreased the IRR of the number of cumulated workday breaks by $14 \%$. The number of prescription renewals was slightly smaller for outpatients than inpatients (figure 3) (online supplementary table 9). However, intervention setting had no impact on the overall number of sick leave renewals. This observation might be explained by the average number of workday breaks per period of sick leaves that

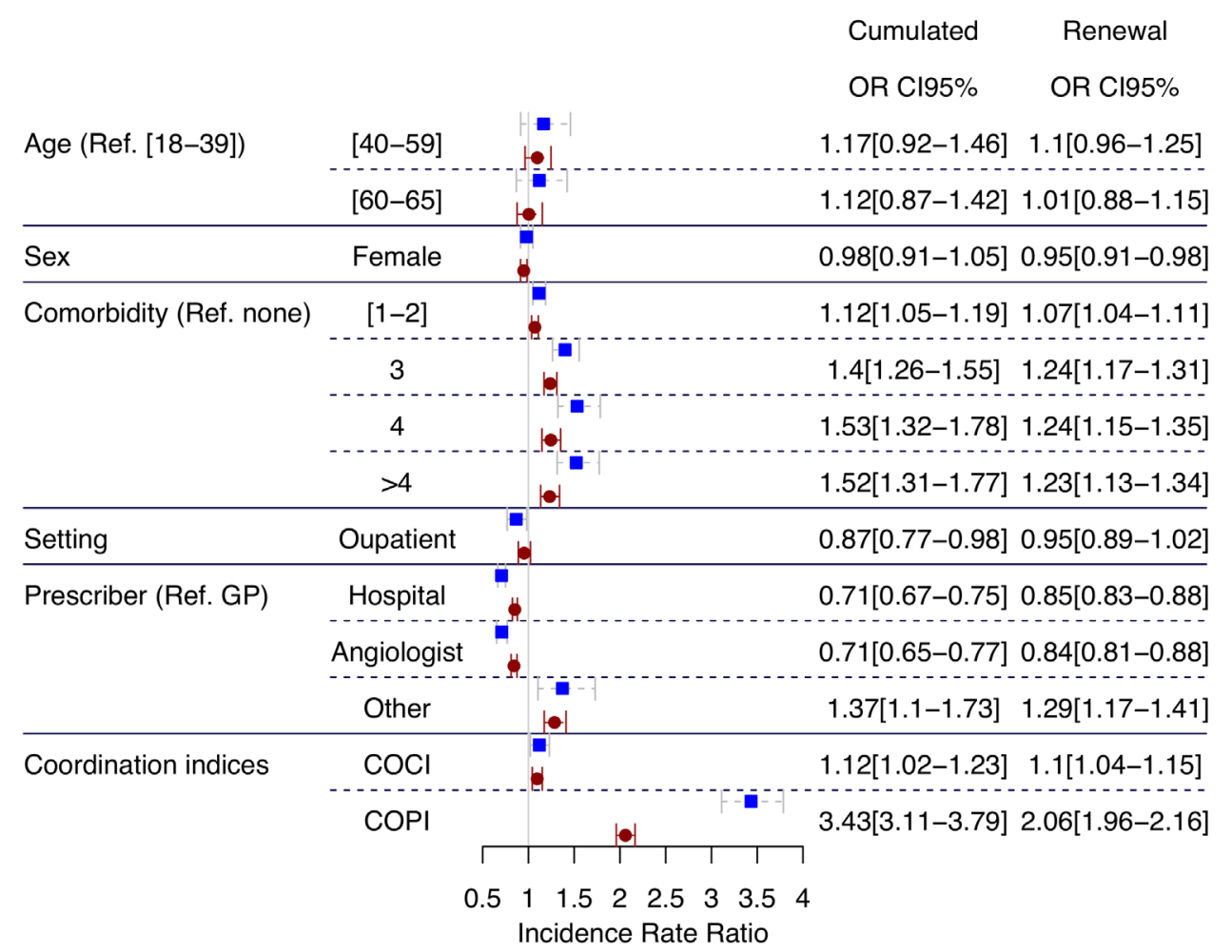

Figure 3 IRRs of cumulated and prescription renewals of sick leave after angioplasty for LEAD intervention with stent placement, in France between 2013 and 2016. COCI, Continuity of Care Index; GP, general practitioner; IRRs, Incidence rate ratios; LEAD, lower extremity arterial diseases. 
is greater for inpatients ( $\mathrm{p}$ value $=0.01$ ) with 6.5 and 5.9 days, respectively.

Compared with prescriptions by a GP, the IRR of the total number of cumulated sick leave days within 180 days of endovascular interventions decreased by $\sim 30 \%$ if prescribed within a hospital or by vascular doctors in medical offices (figure 3). However, it increased by $37 \%$ when prescribed by other specialists in their medical offices. The IRR of the number of sick leave renewals decreased by $15 \%$ and $16 \%$ if prescribed within hospitals or by angiologists in medical offices, respectively, but increased by $27 \%$ if prescribed by other specialists in their medical offices.

The COCI showed that when the degree of coordination between different healthcare providers decreased, the IRR of cumulative days of sick leave increased by $12 \%$ and the number of sick leave renewals increased by $10 \%$. The COPI showed that when the number of sick leave prescriptions delivered by the same group of healthcare practitioners increases, the IRR of cumulated days of sick leaves significantly increased by a factor of 3.43 , and the number of sick leave renewals increased by a factor of 2.06. The principal group of prescribers of sick leave were GPs and other doctors in medical offices with a median of six and three prescriptions per patient, respectively. Interestingly, the COCI and COPI measurements were on average similar whatever the intervention setting (online supplementary table 10)

Overall, except for the coordination indices, the effects of the observed determinants on the number of cumulated days of sick leaves and the renewals of sick leaves after LEAD and varicose vein interventions were similar. Patients' age and sex had no or moderate effects. An increasing trend effect could be observed for comorbidity levels. Outpatients tend to show lower IRR than inpatients. Sick leaves duration and renewals were more important when prescribed by GP than any other health services. The main effect seemed to be coordination that differ between the two pathologies and between settings.

\section{DISCUSSION}

In this analysis of sick leave durations and renewals as proxies of rehabilitation within 180 days of postprocedural follow-up of LEAD and varicose vein interventions according to care settings in France, our findings suggest that outpatient interventions do not induce longer duration of sick leave than inpatient interventions. In fact, our results highlight the benefits of outpatient interventions in decreasing by $13 \%$ the IRR of the cumulated time before resuming work whatever the pathology. Furthermore, the study shows that the variations of practices in the number of cumulated days of sick leave and their renewals prescribed according to the healthcare services are similar for both pathologies. Prescriptions by GPs induced higher numbers of cumulated sick leave days and more renewals. This finding corroborates the importance of coordinating postprocedural follow-up, since the concentration of postintervention care within a single practitioner significantly increases the IRR of duration of sick leave, whatever the pathology. Interestingly, when sick leaves were prescribed by the same group of healthcare providers, the IRR of their duration increased for patients with LEAD but decreased for patients with varicose vein .

To our knowledge, research on the duration of postprocedural rehabilitation process using sick leave prescriptions as proxies for LEAD or varicose vein interventions in inpatient and outpatient settings is scarce. Our findings on the benefits of outpatient interventions for varicose vein are coherent with the work by van Groenendael $e t$ al and the Health Quality Ontario report. ${ }^{13}{ }^{14}$ Although one limitation of our study is the use of sick leave prescriptions and their duration as the only measure to estimate the duration of postprocedural rehabilitation process. Nevertheless, our study describes the contribution of distinct healthcare providers involved in that process while prescribing sick leave. The observed variations in sick leave prescriptions are similar to previous studies based on the French healthcare system where GPs are at the origin of $74 \%$ of the total number of sick leave prescriptions. ${ }^{1516}$ These observations are likely due to the pivotal role of GPs in patient care pathways and an answer to the geographical disparities in the access to healthcare specialists, such as angiologists.

The first strength of our study is the analysis of healthcare consumption for the quasiexhaustive population of LEAD and patients with varicose vein as data were issued from the SNDS, a national medico-administrative database that covers $99 \%$ of the French population. The second strength is the historical depth of our data as our retrospective cohort covers a 4-year period while studies by others were done over a 1-year period. ${ }^{13} 14$

Although representative of the French population, the SNDS has limitations. The database essentially contain data of reimbursed healthcare and associated expenditures but few clinical and sociodemographical information. For instance, adjustment or stratification of the study population by type of work or weekly work load that may be related to post-follow rehabilitation process cannot be done. Similarly, family environment (eg, marital status, offspring) is unknown which is related to intervention setting choice and possible hospital stay conversion from planned same-day discharge to overnight stay. Another limitation is the threshold chosen for the length of stay to select the inpatient population. Although the defined thresholds were based on national guidelines, variations of practices exist at different levels: between hospitals and healthcare professionals, notably. Finally, length of stay in hospital for inpatients were not counted as days off work which may underestimate the benefit of outpatient settings in decreasing sick leave duration and consequently the time to rehabilitation. 


\section{CONCLUSIONS}

Outpatient interventions for LEAD and varicose vein proved to decrease the duration of sick leave compared with conventional intervention. Therefore, outpatient interventions could help saving health insurance money not only associated with hospitals' expenses for bed management but also with work-break payment. In addition, same-day discharge interventions for LEAD have similar timing in postprocedural rehabilitation than for varicose veins. Therefore, duration of sick leave does not seem a determinant of the observed differences in rates of same-day discharge interventions for LEAD and varicose veins. However, the analysis of the coordination indices suggests that healthcare provider coordination is critical for sick leave duration and postprocedural follow-up. Coordination should be specific to the intervention setting and the pathology, especially in the acute care context where postprocedural accessibility to healthcare specialists might be complicated (territorial disparities in travel time and availability). ${ }^{4} 17$ Compared with varicose vein interventions, LEAD interventions require a greater diversity of healthcare providers for postintervention care with tighter coordination. Although we suggest that healthcare coordination after LEAD interventions may not be exclusive to one healthcare specialty, like the GP-centred approach in varicose vein interventions. In addition, in the area of participatory medicine and eHealth, the use of shared electronic health records should be considered seriously by the healthcare providers and users. ${ }^{19-21}$ Though key legal and security challenges are unsolved, ${ }^{22}$ shared electronic health records could become a coordination and shared decision-making tool. ${ }^{23}$ In France, the current promotion of shared e-health records will hopefully be accepted by healthcare services, which should improve care trajectories with enhanced coordination between hospital and office medicine and equity of access to outpatient interventions.

Acknowledgements The authors would like to acknowledge the INDS and CNAMTS for support in accessing the data.

Contributors All four authors were involved in the conception and design of the study. AH and NLM wrote the article with critical revision were by YG and GL. Statistical analysis and interpretation of the data were performed by AH and NLM. All four authors approved the final version of the article.

Funding This work was support by the French Health Products Agency (ANSM) as part of the PEPS platform and the TRACKEDAYS project (TRAjectory of Care for Knowledge on Endovascular DAY Surgery).

Competing interests The authors declare that they have no competing interests.

Patient consent for publication Not required.

Ethics approval Data are issued from the French national health insurance information system with agreement from the French data protection authority (\#1968571). The study protocol was approved by the National Institute of Health Data review board (\#201).

Provenance and peer review Not commissioned; externally peer reviewed.

Data availability statement No data are available.

Open access This is an open access article distributed in accordance with the Creative Commons Attribution Non Commercial (CC BY-NC 4.0) license, which permits others to distribute, remix, adapt, build upon this work non-commercially, and license their derivative works on different terms, provided the original work is properly cited, appropriate credit is given, any changes made indicated, and the use is non-commercial. See: http://creativecommons.org/licenses/by-nc/4.0/.

ORCID iD

Nolwenn Le Meur http://orcid.org/0000-0001-8451-4014

\section{REFERENCES}

1 Lombardi JV, Calligaro KD, Dougherty MJ. Safety and cost savings of endovascular procedures: are outpatient interventions feasible when combined with open surgery? Vasc Endovascular Surg 2002;36:231-5

2 Akopian G, Katz SG. Peripheral angioplasty with same-day discharge in patients with intermittent claudication. J Vasc Surg 2006;44:115-8.

3 Albert B, Davaine J-M, Chaillet M-P, et al. Clinical and economic evaluation of ambulatory endovascular treatment of peripheral arterial occlusive lesions. Ann Vasc Surg 2014;28:137-43.

4 Knutsen Glette M, Kringeland T, Røise O, et al. Hospital physicians' views on discharge and readmission processes: a qualitative study from Norway. BMJ Open 2019;9:e031297.

5 High authority of health. Referencials of work leave prescriptions in five pathologies, 2014. Available: https://www.has-sante.fr/portail/ upload/docs/application/pdf/2014-09/reponse_a_la_saisine_du_10_ mars_2014_en_application_de_larticle_l.161-39_du_code_de_la_ securite_sociale.pdf [Accessed 19 Oct 2018].

6 Tuppin P, de Roquefeuil L, Weill A, et al. French National health insurance information system and the permanent beneficiaries sample. Rev Epidemiol Sante Publique 2010;58:286-90.

7 Bannay A, Chaignot C, Blotière P-O, et al. The best use of the Charlson comorbidity index with electronic health care database to predict mortality. Med Care 2016;54:188-94.

8 Wasey JO. Icd: tools for working with ICD-9 and ICD-10 codes and finding comorbidities. Adv Wound Care 2018.

9 Pollack CE, Hussey PS, Rudin RS, et al. Measuring care continuity: a comparison of Claims-based methods. Med Care 2016;54:e30-4

10 Bice TW, Boxerman SB. A quantitative measure of continuity of care. Med Care 1977;15:347-9.

11 van der Wal WM, Geskus RB. ipw : An R Package for Inverse Probability Weighting. J Stat Softw 2011;43.

12 R Core Team, R Foundation for Statistical Computing. R: a language and environment for statistical computing, 2018. Available: https:// www.R-project.org

13 van Groenendael L, van der Vliet JA, Flinkenflögel L, et al. Treatment of recurrent varicose veins of the great saphenous vein by conventional surgery and endovenous laser ablation. J Vasc Surg 2009;50:1106-13.

14 Medical Advisory Secretariat. Endovascular radiofrequency ablation for varicose veins: an evidence-based analysis. Ont Health Technol Assess Ser 2011;11:1-93.

15 Masson P. La prescription des médecins généralistes : conflits entre la profession médicale et l'Assurance maladie. Sociétés Contemp 2011;83:33-57.

16 Junod W. Prescription and control of work break prescription for disease with regard to medical ethics. Conseil national de l'Ordre des médecins, 2000. http://web.fdn.fr/ amagnouat/metge/IMG/pdf/arret_ travail_ordre.pdf

17 Shahzad M, Upshur R, Donnelly P, et al. A population-based approach to integrated healthcare delivery: a scoping review of clinical care and public health collaboration. BMC Public Health 2019;19:708.

18 Horstman MJ, Mills WL, Herman LI, et al. Patient experience with discharge instructions in postdischarge recovery: a qualitative study. BMJ Open 2017;7:e014842.

19 Abd-Alrazaq AA, Bewick BM, Farragher T, et al. Factors that affect the use of electronic personal health records among patients: a systematic review. Int J Med Inform 2019;126:164-75.

20 Gagnon M-P, Payne-Gagnon J, Breton E, et al. Adoption of electronic personal health records in Canada: perceptions of stakeholders. Int $J$ Health Policy Manag 2016;5:425-33.

21 Seroussi B, Bouaud J. Use of a nationwide personally controlled electronic health record by healthcare professionals and patients: a case study with the French DMP. Stud Health Technol Inform 2017;235:333-7.

22 Christiansen EK, Skipenes E, Hausken MF, et al. Shared electronic health record systems: key legal and security challenges. J Diabetes Sci Technol 2017;11:1234-9.

23 Davis S, Roudsari A, Raworth R, et al. Shared decision-making using personal health record technology: a scoping review at the crossroads. J Am Med Inform Assoc 2017;24:857-66. 